\title{
Effects of array shape and disk ellipticity in dipolar-coupled magnetic metamaterials
}

\author{
Sam D. Slöetjes $\odot,{ }^{1,2,3, *}$ Einar S. Digernes $\odot,{ }^{1}$ Anders Strømberg $\odot,{ }^{1}$ Fredrik K. Olsen, ${ }^{1}$ Ambjørn D. Bang, ${ }^{1}$ \\ Alpha T. N'Diaye, ${ }^{2}$ Rajesh V. Chopdekar $\odot,{ }^{2}$ Erik Folven, ${ }^{1}$ and Jostein K. Grepstad ${ }^{1}$ \\ ${ }^{1}$ Department of Electronic Systems, Norwegian University of Science and Technology (NTNU), NO-7491 Trondheim, Norway \\ ${ }^{2}$ Advanced Light Source, Lawrence Berkeley National Laboratory (LBNL), Berkeley, California 94720, USA \\ ${ }^{3}$ Department of Physics and Astronomy, Uppsala University, Box 516, SE-75120 Uppsala, Sweden
}

(Received 30 April 2021; accepted 29 September 2021; published 21 October 2021)

\begin{abstract}
Two-dimensional lattices of dipolar-coupled thin film ferromagnetic nanodisks give rise to emergent superferromagnetic (SFM) order when the spacing between dots becomes sufficiently small. In this paper, we define micron-sized arrays of permalloy nanodisks arranged on a hexagonal lattice. The arrays were shaped as hexagons, squares, and rectangles to investigate finite-size effects in the SFM domain structure for such arrays. The resulting domain patterns were examined using x-ray magnetic circular dichroism photoemission electron microscopy. At room temperature, we find these SFM metamaterials to be below their blocking temperature. Distinct differences were found in the magnetic switching characteristics of horizontally and vertically oriented rectangular arrays. The results are corroborated by micromagnetic simulations.
\end{abstract}

DOI: 10.1103/PhysRevB.104.134421

\section{INTRODUCTION}

Dipolar-coupled magnetic metamaterials have attracted much attention in the past decade, the most prominent example being artificial spin ice [1]. These metamaterials have successfully facilitated experimental studies on the physics of frustrated spin ice systems, typically found in pyrochlores $[2,3]$, thereby making concepts such as the Coulomb phase more accessible to experimental study [4].

When magnetic moments are arranged on a twodimensional (2D) hexagonal lattice, long-range ferromagnetic order may emerge even in the absence of exchange interactions [5-7]. We have previously shown that it is possible to realize such systems in arrays of monodomain nanoscale disks acting as magnetic dipoles [8]. The possibility to tailor the shape of the individual nanomagnets provides an additional handle for control of the magnetic properties on a microscopic scale. Hexagonal arrays of such magnetic dipoles have been extensively studied theoretically. Politi et al. [9] discussed the long-range order in finite-sized 2D arrays of this lattice symmetry. Jordanovic et al. [10] performed a numerical study on the magnetic domain formation in such arrays, finding domain states like those observed for continuous exchange-coupled 2D micromagnets. Experimentally, Varón et al. [11,12] have investigated the magnetic order in self-assembled aggregates of $15 \mathrm{~nm}$ Co nanoparticles. They found that formation of longitudinal domain walls (DWs) is energetically favored over transverse DWs for rectangular-shaped arrays. Thus, arrays of

\footnotetext{
*samuel.sloetjes@physics.uu.se
}

Published by the American Physical Society under the terms of the Creative Commons Attribution 4.0 International license. Further distribution of this work must maintain attribution to the author(s) and the published article's title, journal citation, and DOI. dipolar-coupled magnets can also be used to engineer artificial ferromagnets.

In this paper, we investigate the magnetic order in finitesized arrays of dipolar-coupled permalloy (Py) nanodisks using $\mathrm{x}$-ray magnetic circular dichroism photoemission electron microscopy (XMCD-PEEM). The arrays are shaped as micron-scale hexagons, squares, and rectangles. At room temperature, we find that the arrays are below their blocking temperature and thus prevented from reaching their magnetic ground state. Such arrays may be suitable candidates for applications that require a pinned magnetization direction, e.g., magnonic crystals.

The magnetization state of the arrays is found to be highly sensitive to minor deviations from circularity of the individual nanodisks [8]. We use micromagnetic simulations that allow for nanomagnet ellipticity, lattice symmetry, and geometric shape of the arrays to reproduce their magnetic switching characteristics.

\section{EXPERIMENTAL}

Using a combination of electron beam lithography, metallization, and lift-off, we prepared arrays of permalloy (Py: $\mathrm{Ni}_{81} \mathrm{Fe}_{19}$ ) disks with diameter $100 \mathrm{~nm}$ and thickness 15 $\mathrm{nm}$ on a silicon substrate. The disks were arranged in a regular hexagonal lattice with $50 \mathrm{~nm}$ spacing. With these dimensions, most of the disks feature monodomain magnetization, producing a significant stray field. Moreover, the $50 \mathrm{~nm}$ spacing ensures a strong dipolar coupling between the disks. The arrays were defined with different geometric shapes, i.e., hexagons, squares, and rectangles, as seen from the scanning electron microscopy (SEM) images in Figs. 1-3. Magnetic domain images were obtained on beamline 11.0.1 (PEEM3) at the Advanced Light Source (ALS), with magnetic contrast derived from taking the difference of the absorption at the $\mathrm{Fe} L_{3}$ edge for left- and right-handed circularly polarized 

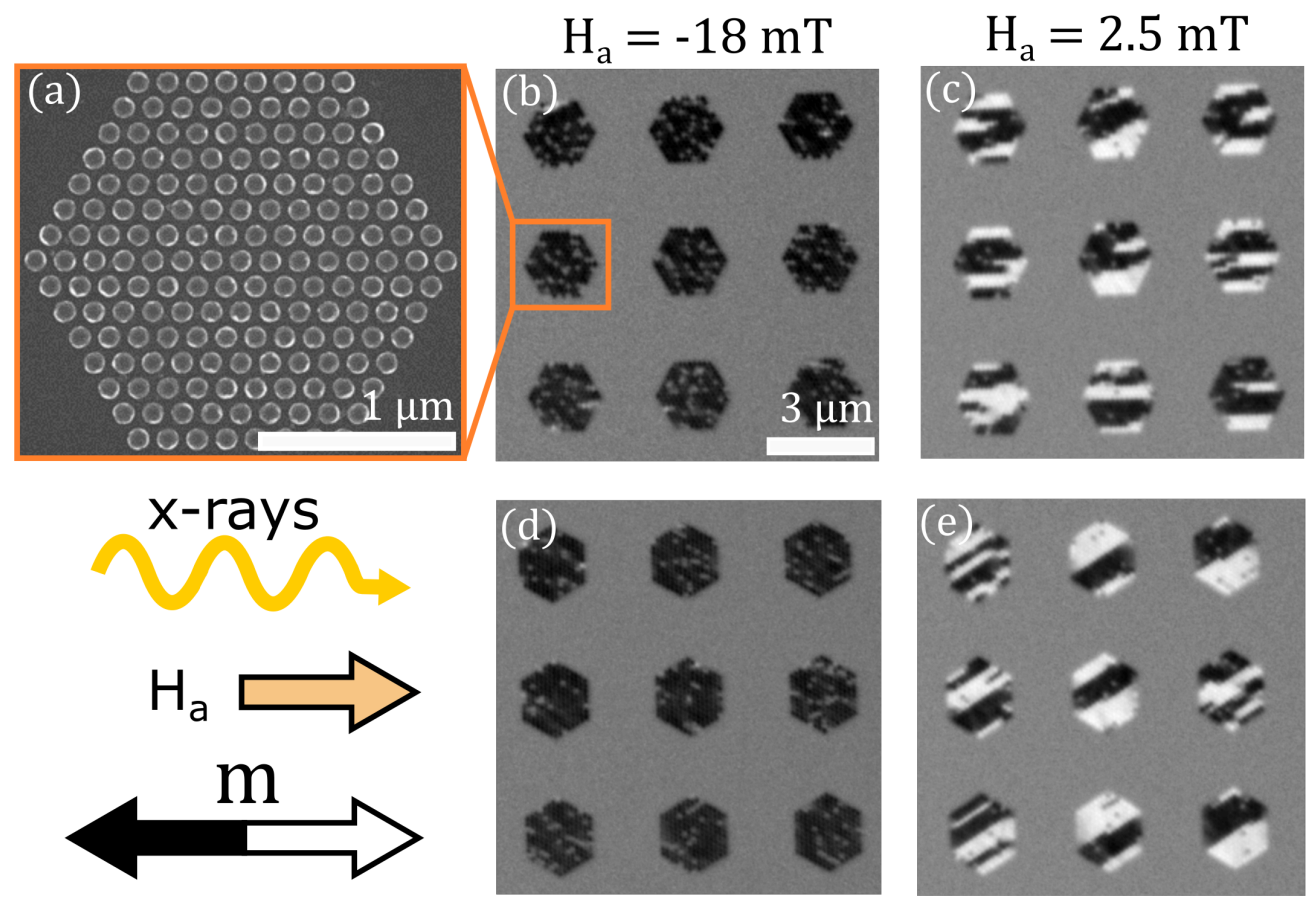

FIG. 1. (a) Scanning electron microscopy (SEM) image of the hexagon-shaped (hexagonal lattice) array. (b) and (d) X-ray magnetic circular dichroism photoemission electron microscopy (XMCD-PEEM) images of such arrays after magnetic saturation and (c) and (e) after applying a small field in the opposite direction, with X-ray incidence, applied field $H_{a}$, and maximum XMCD contrast, as indicated by the inset arrows.

$\mathrm{x}$ rays, respectively [13]. Since XMCD-PEEM does not permit an applied magnetic field during measurement, the magnetic domain images in Figs. 1-3 were all recorded in remanence, i.e., after removal of the applied magnetic field pulse $\left(H_{a}\right)$. In this experiment, all measurements were carried out at room temperature $(T=295 \mathrm{~K})$.

\section{RESULTS AND DISCUSSION}

Figure 1 shows results from XMCD-PEEM measurements of the hexagon-shaped arrays. Each array consists of 15 magnets along the diagonal, measuring $2.25 \mu \mathrm{m}$. The hexagons were defined in two distinct orientations, one with a pair of edges parallel to the horizontal ( $x$ direction), cf. Figs. 1(b) and $1(\mathrm{c})$, and the other with two edges parallel to the vertical
( $y$ direction), cf. Figs. 1(d) and 1(e). In the following, these will be referred to as the horizontal and vertical orientation, respectively. Initially, we used a magnetic field of $18 \mathrm{mT}$ to saturate the magnets in the $-x$ direction. The uniform black contrast observed in Figs. 1(b) and 1(d) indicates that the nanomagnets are below their blocking temperature and oriented parallel to the applied magnetic pulse direction. In addition to the predominant black contrast, we observe several gray dots inside these magnetic structures, which can most likely be attributed to nanodisks with flux-closure magnetization, as nanomagnets of this dimension are at the limit of single-domain magnetization [14]. After saturating the arrays, we applied a small field of $2.5 \mathrm{mT}$ in the opposite $(+x)$ direction and examined the resulting magnetization patterns, cf. Figs. 1(c) and 1(e). We observe a domain pattern with
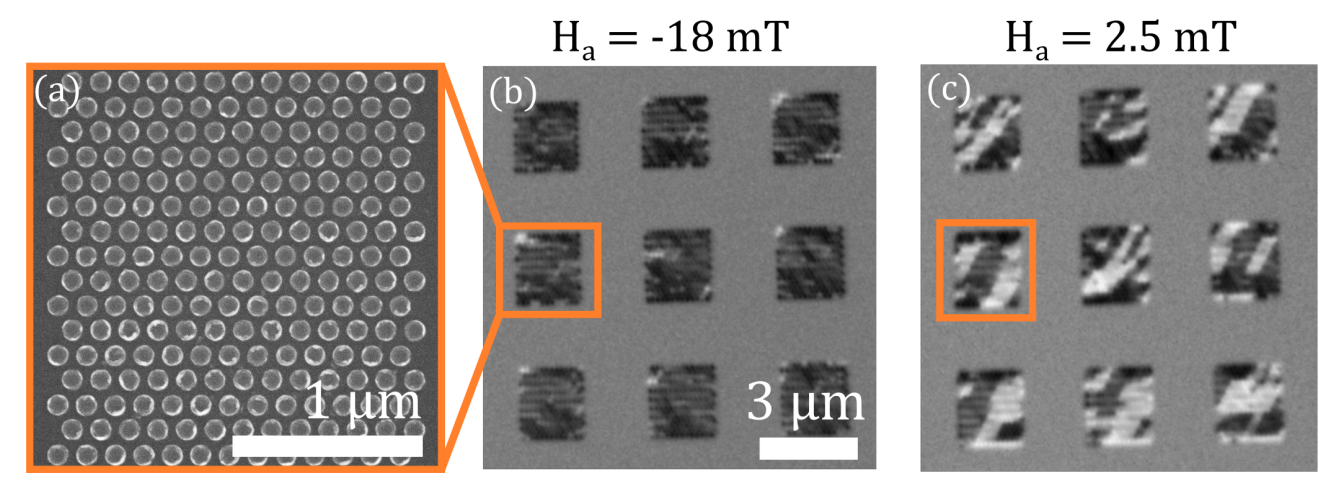

FIG. 2. (a) Scanning electron microscopy (SEM) image of the square (hexagonal lattice) array. Note the jagged vertical edges. (b) X-ray magnetic circular dichroism photoemission electron microscopy (XMCD-PEEM) images of such arrays after saturation and (c) after applying a small field in the opposite direction. 

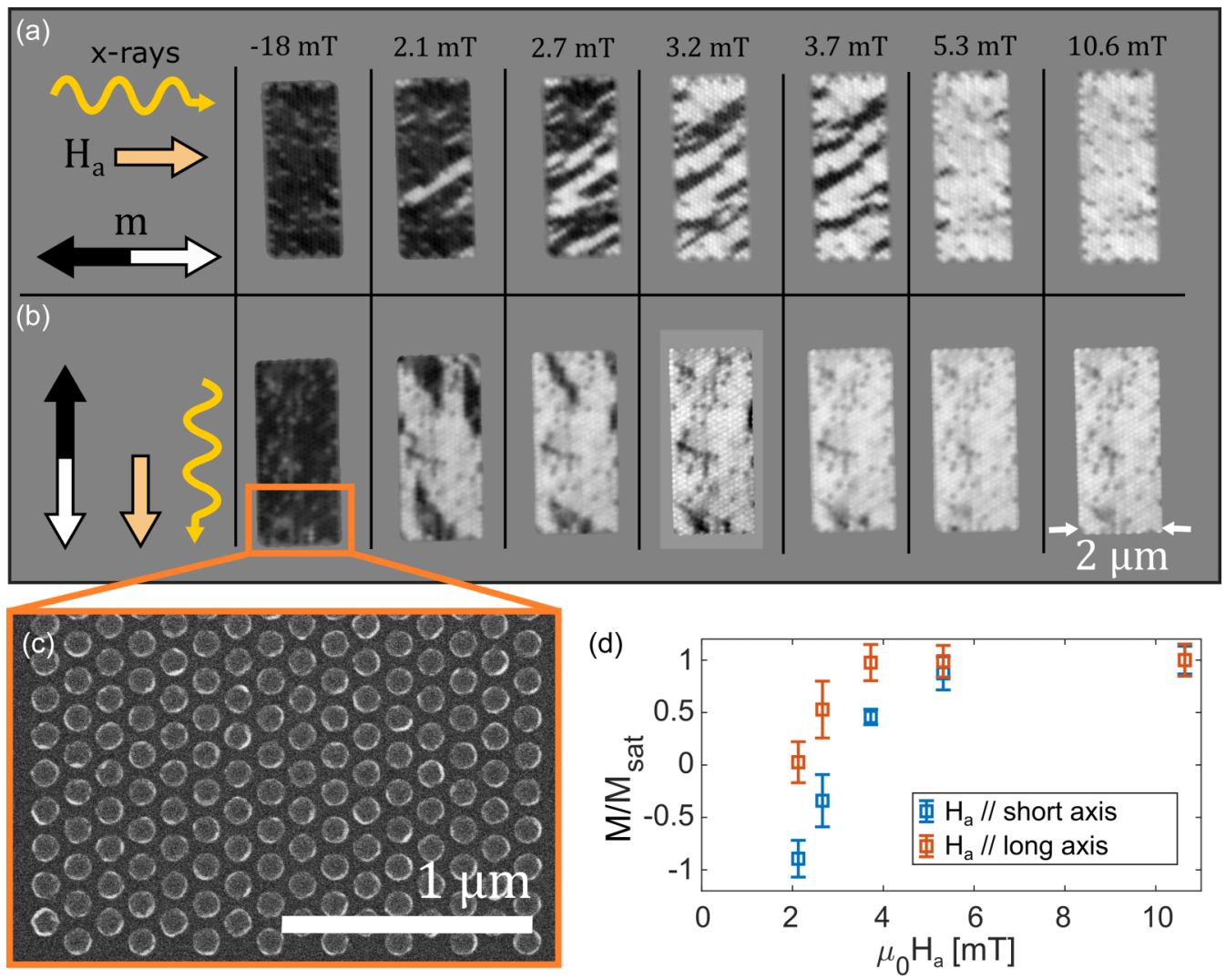

FIG. 3. Switching series for a rectangular-shaped array of nanomagnets with (a) $H_{a}$ perpendicular and (b) $H_{a}$ parallel to the long edge of the rectangle. (c) Scanning electron microscopy (SEM) image of a section of the hexagonal array. (d) Graph showing $M$ vs $H_{a}$ for the switching series.

extended regions of uniform magnetization after the reversing field pulse, suggesting superferromagnetic (SFM) order in the array.

For horizontal hexagons, we note the presence of domains with two distinct magnetization directions, indicated by the mostly black and white contrast and no gray contrast. These domains are separated by DWs that are for the most part horizontal. We note that this is consistent with DW alignment along a principal axis of the lattice. For vertical hexagons, we observe a pronounced preference for formation of DWs along the direction canted $30^{\circ}$ from the applied magnetic field axis. A shape analysis based on the SEM images of these arrays shows that the nanodisks have an average ellipticity of $4 \%$, resulting in a shape-induced anisotropy. The long axis of this elliptic distortion forms on average an angle of $\sim 15^{\circ}$ with the horizontal axis. The principal lattice directions of these arrays are $30^{\circ}, 90^{\circ}$, and $150^{\circ}$ with the horizontal. As such, the preferred direction for DW formation resulting from the shape anisotropy of the nanodisks and the lattice symmetry is $30^{\circ}$.

In Fig. 2, we show results for a $2 \times 2 \mu \mathrm{m}$ square array of hexagonally ordered nanodisks. Fitting a hexagonal lattice to a square array necessarily leads to a jagged termination of two opposite edges, as observed from the SEM image in Fig. 2(a). After saturation in an applied field pulse of $18 \mathrm{mT}$, the arrays are predominantly magnetized in the $-x$ direction, cf. Fig. 2(b). After applying a field of $+2.5 \mathrm{mT}$ in the direction opposite that of the initial saturation, the magnetization of the arrays forms SFM domain patterns resembling those of continuous thin film micromagnets. For example, the domain pattern of the highlighted array in Fig. 2(c) resembles the Z-type domain state previously recorded for square thin film micromagnets $[15,16]$.

Figure 3 shows XMCD-PEEM images of two $2 \times 5 \mu \mathrm{m}$ rectangular arrays. The array in Fig. 3(a) is aligned with the long axis perpendicular to the applied field, and the array in Fig. 3(b) is aligned with the long axis parallel to the applied field. For the field applied perpendicular to the long axis, we observe a gradual switching of the array magnetization with increasing field strength. SFM domains are formed with DWs inclined $\sim 30^{\circ}$ with respect to the horizontal edges of the rectangle. This $30^{\circ}$ inclination of the DWs coincides with a principle axis of the hexagonal lattice. However, the lattice symmetry does not explain the apparent preference for DW alignment along only one of the two equivalent directions $+30^{\circ}$ and $-30^{\circ}$. As discussed above for hexagonal arrays, we attribute the $+30^{\circ}$ preference in Fig. 3(a) to the nanodisk ellipticity in these arrays, for which the average long axis orientation is $+15^{\circ}$, as determined from the SEM images.

Figure 3(b) shows switching of the array magnetization with the field applied parallel to the long edge. At $\mu_{0} H_{a}=$ $2.1 \mathrm{mT},>60 \%$ of the nanodisks have switched as compared with $10 \%$ with the field applied perpendicular to the long edge. In these magnetic metamaterials, switching of the array magnetization will also depend on factors beyond the orientation of the switching field, such as ellipticity of the individual disks and orientation of the hexagonal lattice with respect to 


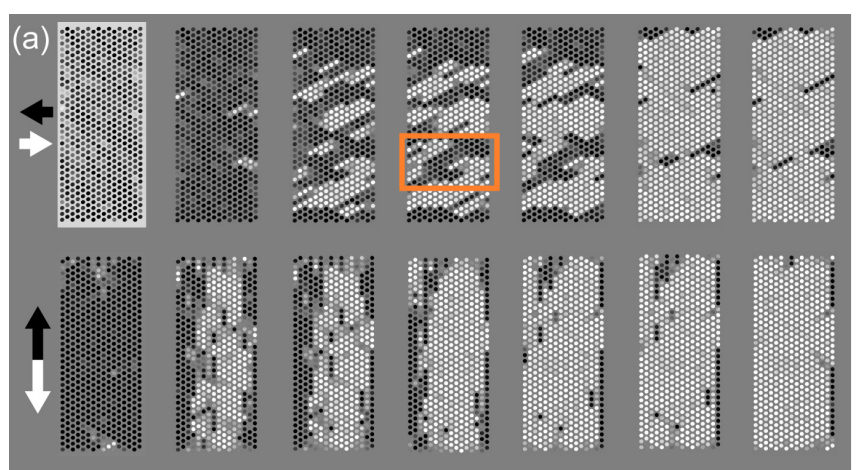

(b)
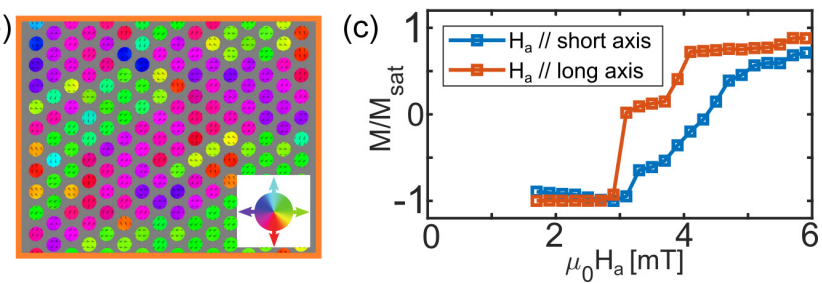

FIG. 4. (a) Simulated switching for rectangular arrays of nanodisks with hexagonal lattice symmetry with the switching field applied perpendicular (top row) and parallel (bottom row) to the long axis of the rectangle. (b) Zoom-in on the region marked with a dashed frame in (a). (c) $M$ vs $H_{a}$ graphs showing the difference in switching behavior for the two directions of the applied field.

the applied field. Analysis of the SEM images shows a small deviation from circularity of $4 \%$. To investigate the differences in switching for normal and parallel orientation of the applied field with respect to the long axis of the rectangles, we performed micromagnetic simulations using the simulation package MUMAX3 [17].

As input material parameters for the Py disks, we used an exchange stiffness of $A_{\mathrm{ex}}=1 \times 10^{-11} \mathrm{~J} / \mathrm{m}$ and a saturation magnetization of $M_{\text {sat }}=3 \times 10^{5} \mathrm{~A} / \mathrm{m}$. This low value for $M_{\text {sat }}$ was found to provide the best correspondence to the experimental data and may be attributed to partial oxidation of the Py nanomagnets. A biaxial anisotropy of $K_{1}=$ $3000 \pm 300 \mathrm{~J} / \mathrm{m}^{3}$ with random in-plane orientation was included to account for pinning of the magnetic moments of the individual disks below the blocking temperature. In the simulations, we chose a fine grid for the in-plane directions $l_{x} \times l_{y}=2.6 \times 2.2 \mathrm{~nm}$ and a single cell for the out-of-plane direction $l_{z}=15 \mathrm{~nm}$ to reduce the effects of anisotropy originating from projecting circular particles onto a square grid. Finally, we included an elliptic distortion of the disk elements, consistent with that measured from the SEM images.

The outcome of these simulations is displayed in Fig. 4(a), showing close correspondence with the experimental data. In Fig. 4(b), we call attention to the region marked with an orange frame in the top row of rectangular arrays in Fig. 4(a). The SFM magnetization texture consists of domains where each domain is comprised of tens of disks. In these domains, most disks have their magnetic moments inclined $\sim 30^{\circ}$ with the horizontal axis. In Fig. 4(c), we have plotted the $M$ vs $H_{a}$ graphs obtained upon switching for both orientations of the rectangles. We note that the domain patterns of these micromagnetic simulations are in good correspondence with the experimental data.
TABLE I. Demagnetizing factors for circular and $4 \%$ elliptical uncoupled disks and for metapatterned arrays with circular disks.

\begin{tabular}{lccc}
\hline \hline & $N_{x}$ & $N_{y}$ & $N_{z}$ \\
\hline Circular disk & 0.1338 & 0.1338 & 0.7371 \\
Disk with 4\% ellipticity & 0.1284 & 0.1349 & 0.7364 \\
Hexagon array & 0.1187 & 0.1189 & 0.7649 \\
Square array & 0.1185 & 0.1189 & 0.7644 \\
Rectangular array & 0.1120 & 0.1144 & 0.7568 \\
\hline \hline
\end{tabular}

To assess the effects of nanomagnet ellipticity and array geometry on the magnetic anisotropy, we computed the effective demagnetizing factors $N_{x}, N_{y}$, and $N_{z}$ for a magnet with $4 \%$ deviation from circularity as well as for the different metapatterned arrays in the absence of ellipiticity. To this end, we used the relation $N_{i}=2 E_{\mathrm{demag}} / V \mu_{0} M_{s}^{2}$, where $V$ is the volume of magnetic material and $E_{\text {demag }}$ the demagnetization energy. The demagnetization energy was found from micromagnetic simulations in which the magnetization was saturated in the $x, y$, and $z$ directions, respectively. Here, the $x$ direction coincides with the long axis of the elliptical magnet and with the horizontal (vertical) direction in the metapatterned arrays shown in Figs. 1 and 2 (Figs. 3 and 4). The demagnetization factors for circular and $4 \%$ elliptical uncoupled disks as well as for metapatterned arrays with circular disks are shown in Table I.

We find that a $4 \%$ disk ellipticity has a significantly stronger impact on the demagnetizing factors $\left(\frac{\Delta N_{x, y}}{N_{x}} \approx 5 \%\right)$ and thus the magnetic anisotropy than the metapatterned array geometry. The largest impact of the metapattern shape is found for the rectangular array $\left(\frac{\Delta N_{x, y}}{N_{x}} \approx 2.1 \%\right)$. This analysis of demagnetizing factors confirms our conjecture that the shape of the individual nanomagnets constitutes the dominant contribution to magnetic anisotropy. The demagnetizing factors for the square- and hexagon-shaped arrays both are within the error margins of the calculation, thus indicating a negligible contribution to the anisotropy. Moreover, we note that the computed values for $N_{x, y}$ of a single disk differ appreciably from those of the rectangular- and hexagonshaped arrays. We attribute this finding to the fact that the arrays consist of large numbers of nanomagnets, thus increasing the aspect ratio between the in- and out-of-plane dimensions.

\section{CONCLUSIONS}

We have investigated the magnetic properties of SFM ensembles of Py thin film nanodisks arranged in a hexagonal lattice for finite-sized arrays of different shapes. It was found that the direction of magnetization in these arrays is primarily determined by the lattice geometry and strongly affected by deviations from perfect circularity of the nanodisk magnetic elements, whereas the actual geometric shape of the array is of minor importance, at least in the presence of a minor disk anisotropy. When measuring the magnetic switching of rectangular arrays, we found that these arrays switch more gradually and at higher threshold fields with the field applied perpendicular to the long edges of the rectangle compared 
with switching with the field applied parallel to these edges. Micromagnetic simulations confirm this behavior, with good correspondence to the orientation and density of superdomains and super-DWs.

\section{ACKNOWLEDGMENTS}

This paper used resources of the ALS, which is a U.S. Department of Energy Office of Science User Facility under Contract No. DE-AC02-05CH11231. S.D.S. acknowledges support from the ALS Doctoral Fellowship in Residence. Partial funding was obtained from the European Union's Horizon 2020 FET-Open program under Grant Agreement No. 861618 (SpinENGINE) and the Norwegian Ph.D. Network on Nanotechnology for Microsystems, which is sponsored by the Research Council of Norway, Division for Science, under Contract No. 221860/F60. The Research Council of Norway is acknowledged for support of the Norwegian Microand Nanofabrication Facility, NorFab, under Contract No. 245963/F50.
[1] R. F. Wang, C. Nisoli, R. S. Freitas, J. Li, W. McConville, B. J. Cooley, M. S. Lund, N. Samarth, C. Leighton, V. H. Crespi, and P. Schiffer, Artificial 'spin ice' in a geometrically frustrated lattice of nanoscale ferromagnetic islands, Nature (London) 439, 303 (2006).

[2] S. T. Bramwell and M. J. P. Gingras, Spin ice state in frustrated magnetic pyrochlore materials, Science 294, 1495 (2001).

[3] M. J. Harris, S. T. Bramwell, D. F. McMorrow, T. Zeiske, and K. W. Godfrey, Geometrical Frustration in the Ferromagnetic Pyrochlore $\mathrm{Ho}_{2} \mathrm{Ti}_{2} \mathrm{O}_{7}$, Phys. Rev. Lett. 79, 2554 (1997).

[4] Y. Perrin, B. Canals, and N. Rougemaille, Extensive degeneracy, Coulomb phase and magnetic monopoles in artificial square ice, Nature (London) 540, 410 (2016).

[5] J. M. Luttinger and L. Tisza, Theory of dipole interaction in crystals, Phys. Rev. 70, 954 (1946).

[6] P. Politi and M. G. Pini, Dipolar interaction between twodimensional magnetic particles, Phys. Rev. B 66, 214414 (2002).

[7] S. D. Slöetjes, H. H. Urdahl, J. K. Grepstad, and E. Folven, Tailoring the magnetic order in a supermagnetic metamaterial, AIP Adv. 7, 056325 (2017).

[8] E. Digernes, S. D. Slöetjes, A. Strømberg, A. D. Bang, F. K. Olsen, E. Arenholz, R. V. Chopdekar E., J. K. Grepstad, and E. Folven, Direct imaging of long-range ferromagnetic and antiferromagnetic order in a dipolar metamaterial, Phys. Rev. Res. 2, 013222 (2020).

[9] P. Politi, M. G. Pini, and R. L. Stamps, Dipolar ground state of planar spins on triangular lattices, Phys. Rev. B 73, 020405(R) (2006).
[10] J. Jordanovic, M. Beleggia, J. Schiøtz, and C. Frandsen, Simulations of super-structure domain walls in two-dimensional assemblies of magnetic nanoparticles, J. Appl. Phys. 118, 043901 (2015).

[11] M. Varón, M. Beleggia, J. Jordanovic, J. Schiøtz, T. Kasama, V. F. Puntes, and C. Frandsen, Longitudinal domain wall formation in elongated assemblies of ferromagnetic nanoparticles, Sci. Rep. 5, 14536 (2015).

[12] M. Varón, M. Beleggia, T. Kasama, R. J. Harrison, R. E. DuninBorkowski, V. F. Puntes, and C. Frandsen, Dipolar magnetism in ordered and disordered low-dimensional nanoparticle assemblies, Sci. Rep. 3, 1234 (2013).

[13] S. Anders, H. A. Padmore, R. M. Duarte, T. Renner, T. Stammler, A. Scholl, M. R. Scheinfein, J. Stöhr, L. Séve, and B. Sinkovic, Photoemission electron microscope for the study of magnetic materials, Rev. Sci. Instrum. 70, 3973 (1999).

[14] R. P. Cowburn, D. K. Koltsov, A. O. Adeyeye, M. E. Welland, and D. M. Tricker, Single-Domain Circular Nanomagnets, Phys. Rev. Lett. 83, 1042 (1999).

[15] Y. Takamura, E. Folven, J. B. R. Shu, K. R. Lukes, B. Li, A. Scholl, A. T. Young, S. T. Retterer, T. Tybell, and J. K. Grepstad, Spin-Flop Coupling and Exchange Bias in Embedded Complex Oxide Micromagnets, Phys. Rev. Lett. 111, 107201 (2013).

[16] S. D. Sloetjes, E. Digernes, F. K. Olsen, R. V. Chopdekar, S. T. Retterer, E. Folven, and J. K. Grepstad, Interplay between bulk and edge-bound topological defects in a square micromagnet, Appl. Phys. Lett. 112, 042401 (2018).

[17] A. Vansteenkiste, J. Leliaert, M. Dvornik, M. Helsen, F. GarciaSanchez, and B. Van Waeyenberge, The design and verification of MuMaX3, AIP Adv. 4, 107133 (2014). 